$\xi=$

\title{
A two-phase model for the aqueous outflow through the trabecular meshwork
}

\author{
Ram Avtar, Swati Srivastava* \\ Department of Mathematics, Harcourt Butler Technological Institute, Kanpur, India \\ *Corresponding author E-mail:feb13swati@gmail.com
}

\begin{abstract}
A two-phase steady-state model for the percolation of aqueous humor through the trabecular meshwork (TM) in eye has been developed. The model treats the meshwork as an annular porous cylinder comprised of two concentric rings that represent the uveal-corneoscleral meshwork and juxtacanalicular meshwork. Both the rings are assumed to be made up of homogeneous, isotropic, viscoelastic material swollen with continuously flowing aqueous humor through the tissue with different structural properties. The model incorporates a strain-dependent permeability function. An analytical solution to the mathematical model has been obtained and the expressions for the displacement and fluid pressure distributions have been derived. The computational results for the displacement in solid phase, the fluid pressure distribution and the dilatation of the ocular tissue material have been presented through the graphs. The effects of structural model parameters on the displacement and the dilatation have also been investigated.
\end{abstract}

Keywords:Aqueous Humor; Trabecular Meshwork; Uveal-Corneoscleral Meshwork; Juxtacanalicular Meshwork.

\section{Introduction}

Vision loss in most forms of glaucoma is related to elevated intraocular pressure (IOP) with subsequent injury to the optic nerve. In humans, $75 \%$ of the resistance to the aqueous humor outflow is localized in the TM. The major site of resistance within the TM structure has not yet been well characterized. It has been suggested by some studies $[6,22]$ that it resides in the juxtacanalicular matrix. A basic concept that impairment in/obstruction to the aqueous humor outflow and its subsequent accumulation in the anterior chamber results in elevation of IOP which is a characteristic of open-angle glaucoma, is a central tenet in glaucoma pathology and treatment. The most of current therapeutic procedures to treat glaucoma seek to lower IOP by decreasing aqueous humor outflow resistance residing in the TM (conventional) pathway, and are, therefore, directed towards the increasing aqueous outflow by opening existing drainage pathways or by creating new flow pathways or by some mechanisms that are not completely clear. Thus, identifying the principle site of the outflow resistance in the trabecular meshwork pathway and understanding the complex mechanisms that regulate aqueous humor outflow resistances is essential for improved management of glaucoma.

The TM is one of the main ocular structures related to aqueous humor dynamics and one of the principal locations of aqueous outflow. It is a triangular porous structure in cross-section that consists of connective tissue surrounded by endothelium. TM can be divided in three components: uveal meshwork (UM), corneoscleral meshwork (CSM) and juxtacanalicular meshwork (JCM). The UM forms the lateral border of the anterior chamber. It consists of bands of connective tissue with irregular openings that measure between 25 to $75 \mu \mathrm{m}$. The CSM is the most extensive portion of the TM. It is composed of perforated sheets that become progressively smaller nearing SC. It is organized into four concen- tric layers viz. from within outwards connective tissue with collagen fiber layer, elastic fiber layer, "glass membrane" layer and endothelial layer. The outermost part of the TM, composed of a layer of connective tissue lined on either side by endothelium, is called the JCM. JCM is believed to be the source of majority of the outflow resistance. The central connective tissue layer has variable thickness and is non-fenestrated and the outer endothelial layer comprises the inner wall of canal of Schlemm. The uveal and corneoscleral meshwork, that make up the trabecular meshwork, are highly porous structures with numerous openings that range in size from 25-75 $\mu \mathrm{m}$ in the proximal regions of the uveal meshwork to 2-15 $\mu \mathrm{m}$ in the deeper layers of the corneoscleral meshwork.

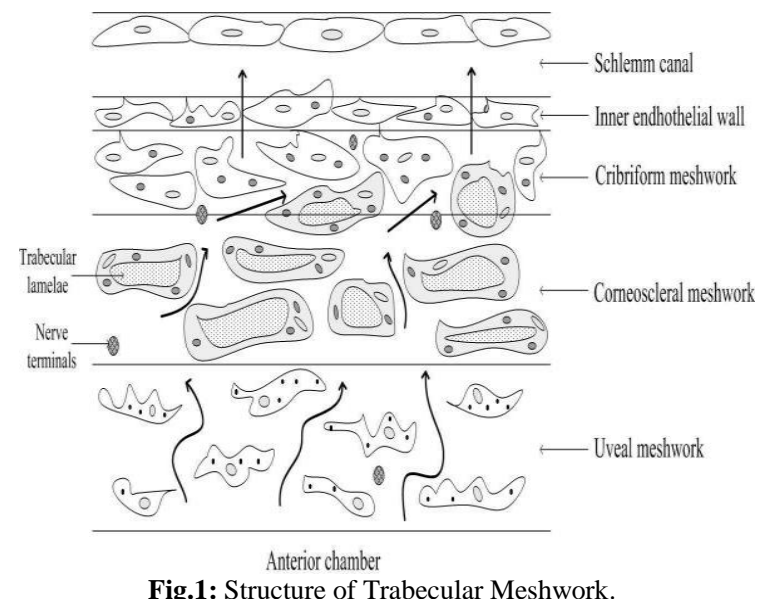

In addition to the experimental investigations of the aqueous flow phenomenon, the modeling and simulation of the outflow of $\mathrm{AH}$ through the TM, may contribute to the elucidation of the mechan- 
ism of outflow resistance increase in normal and glaucomatous eyes. In view of this, there is a need to develop more realistic mathematical models for the outflow of AH through the TM and simulate the outflow phenomenon. Poiseuille flow [15] models as well as various empirical models have been applied to describe aqueous flow through the trabecular meshwork. The concept of the convectional aqueous outflow pathway was modeled as tubes with leaky walls and a network of electrical resistances by Moses et al. [18]. Ethier et al. [6] developed a model based on the analysis of the effect of gel-concentration on the JCM resistance to outflow, but they did not focus on the flow field and related IOP. Tandon and Avtar [23] presented a biphasic continuum model for aqueous flow through the meshwork and concluded that the IOP has a permeability-decreasing effect, whereas the elasticity of the meshwork shows a permeability-increasing effect. A simple mathematical model of aqueous outflow through the trabecular meshwork to provide an estimate of effect of IOP on the flow characteristics of the aqueous humor proposed by Avtar and Srivastava [3].

The work presented in this paper is concerned with the development of a two-phase model for the aqueous humor outflow through the trabecular meshwork. The TM is modeled as an annular cylinder and is comprised of two concentric rings that represent uveal-corneoscleral meshwork and juxtacanalicular meshwork. Analytical solution to the mathematical model has been obtained and the expressions for flow-characteristics have been derived The computational results have been presented through graphs. In the previous models, the TM has been represented as a single annular region of homogenous matrix and effects of the structural parameters have not been investigated. In our model, the TM is treated as an annular cylinder with two adjoining rings of different mechanical and hydraulic properties.

\section{Model development}

The TM is represented as an annular cylinder with a large central cavity which represents the anterior chamber (AC). The annular region is split into two adjoining rings. The outer annular region corresponds to the juxtacanalicular meshwork, the inner annular region to the uveal-corneoscleral meshwork. The two regions mechanically interact at their interface. The uveal-corneoscleral meshwork and juxtacanalicular meshwork of the TM are modeled as a two-phase medium composed of a porous elastic matrix saturated with aqueous fluid.

IOP is the loading force to which the outflow system normally responds under the action of an external load (IOP). The deformation of TM treated as porous solid filled with fluid is accompanied by fluid flow through porous medium. The model of TM is developed by incorporating the coupled dynamical viscous interaction between the fluid flow and the mechanical deformation of the TM tissue.

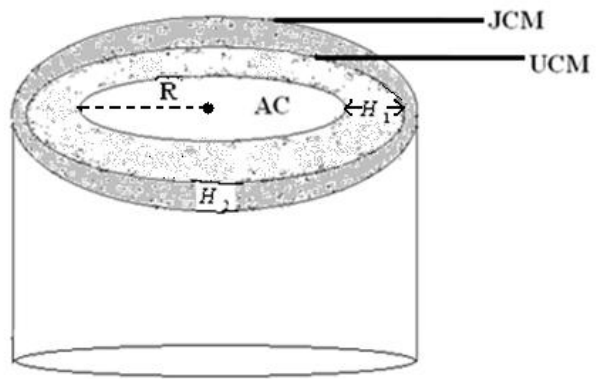

Fig. 2: Proposed Schematic Model of Trabecular Meshwork.

\subsection{Assumptions}

The TM is comprised of two different isotropic, homogenous, porous, deformable matrices swollen with continuously flowing aqueous humor. The permeability of the meshwork is a function of the dilatation. Only the fluid mechanical aspects of aqueous flow phenomenon are considered. Both the constituents of the system are isothermal. The aqueous fluid and solid matrix are intrinsically incompressible. Inertial and body forces are neglected and diffusional couples do not exist. The displacement of the tissue depends only on radial position. Aqueous flow is steady, slow, laminar, Newtonian, viscous and incompressible. The protein accumulation, the mascular contraction, the nervous control and the cilliary muscle traction are neglected. Due to the fluid motion in the lumen, the axial wall shear stress is negligible in comparison to other stresses acting on the wall.

\subsection{Governing equations}

The balances of mass and linear momentum over a small volume element of a saturated porous material undergoing deformation due to fluid flow results in the following equations for each phase $\varepsilon(\varepsilon=s$ for solid and $=f$ for fluid $)[4,11]$.

$\frac{\partial\left(n^{\varepsilon} \rho^{\varepsilon}\right)}{\partial t}+\nabla \cdot\left(n^{\varepsilon} \rho^{\varepsilon} v^{\varepsilon}\right)=m^{\varepsilon}$

$\frac{\partial\left(n^{\varepsilon} \rho^{\varepsilon} v^{\varepsilon}\right)}{\partial t}+\nabla \cdot\left(n^{\varepsilon} \rho^{\varepsilon} v^{\varepsilon} v^{\varepsilon}\right)-\nabla \cdot \sigma^{\varepsilon}-n^{\varepsilon} \rho^{\varepsilon} \boldsymbol{b}^{\varepsilon}=\boldsymbol{R}^{\varepsilon}$,

where $n^{\varepsilon}, \rho^{\varepsilon}, v^{\varepsilon}$ are volume fraction, intrinsic mass density, and velocity of phase $\varepsilon$ respectively. $\sigma^{\varepsilon}$ and $\boldsymbol{b}^{\varepsilon}$ denote stress tensor and density of external body forces. $m^{\varepsilon}$ and $\boldsymbol{R}^{\varepsilon}$ are rate of mass and linear momentum exchange between the two phases. Besides, for a closed mass system, the equations must satisfy the relations:

$m^{s}+m^{f}=0$,

$\boldsymbol{R}^{s}+\boldsymbol{R}^{f}=0$

In view of the assumptions: the steady-state flow behavior, the negligible inertial forces, no mass exchange allowed between phases, negligible/zero solid velocity and a constant intrinsic density of the fluid, the system of four balance eqns. (1) and (2) are simplified and rearranged using conditions (3) and (4) to the following steady-state form:

$\nabla \cdot q=0$,

$\nabla \cdot\left(\sigma^{s}+\sigma^{f}\right)=0$

$\nabla \cdot \sigma^{f}+R^{f}=0$

where $q=n^{f} v^{f}$ is the rate of volumetric flow.

The stress state, for small deformations of an isotropic material, is described by the equations [1].

$$
\begin{aligned}
& \sigma^{f}=-n p \boldsymbol{I}, \\
& \sigma_{v}^{\prime}=\sigma^{s}+\sigma^{f}+p \boldsymbol{I}=2 \mu \boldsymbol{e}+\lambda t r \boldsymbol{e} \boldsymbol{I},
\end{aligned}
$$

where $n\left(=n^{f}\right)$ is the porosity of the material, $\mathrm{p}$ the pore fluid pressure, $\sigma_{v}^{\prime}$ is called the effective stress, $\mu$ and $\lambda$ are the Lame constants of the meshwork, and the small strain tensor e is related to the displacement of the solid matrix, $u$ by

$\boldsymbol{e}=\frac{1}{2}\left[\nabla \boldsymbol{u}+(\nabla \boldsymbol{u})^{T}\right]$ 
The interaction force, $\boldsymbol{R}^{f}$ for the steady flow of fluid through porous material [4] is given by

$\boldsymbol{R}^{f}=-\frac{\mu^{f} n}{k} \boldsymbol{q}+p \nabla n$,

where $\mu^{f}$ is the dynamic viscosity of the fluid, and $\mathrm{k}$, the intrinsic permeability of the solid. In R.H.S., the first term represents viscous interaction of fluid with solid matrix, and the second term the buoyancy force due to the variable volume fraction of phases. Following studies [13] we utilize the following relationship between the permeability of the meshwork material and strain

$k^{-1}=k_{0}^{-1}(1-M$ tre $)$,

where $k_{0}$ is the permeability and $\mathrm{M}$ is the material constant of the trabecular meshwork and tr e is the dilatation which, in cylindrical coordinates, is given by

$\operatorname{tr} \boldsymbol{e}=\frac{d u}{d r}+\frac{u}{r}$.

This expression is a small-strain approximation to $k=k_{0} \exp (M$ tr $e$ ) proposed by Lai and Mow [13] for cartilage.

Using the constitutive functions defined by relations (8)-(13) and assuming the dependence of the fluid velocity, fluid pressure and solid displacement on the radial coordinates only, equations (5)(7) are reduced to the following system of equations:

$\frac{d q}{d r}+\frac{q}{r}=0$

$(2 \mu+\lambda)\left(\frac{d^{2} u}{d r^{2}}+\frac{1}{r} \frac{d u}{d r}-\frac{u}{r^{2}}\right)-\frac{d p}{d r}=0$,

$\frac{d p}{d r}+\frac{\mu^{f}}{k_{0}}\left[1-M\left(\frac{d u}{d r}+\frac{u}{r}\right)\right] q=0$.

\subsection{Boundary and matching conditions}

The effective stress at the inner (anterior chamber) surface of the ring is prescribed equal to zero and the displacement of the solid and the fluid pressure at the outer boundary is assumed equal to zero and discharge velocity of the fluid is prescribed as a constant Q.

$$
\begin{aligned}
& \text { i) } \quad \sigma_{v}^{\prime}=0 \\
& \text { for } r=R \text {, } \\
& \text { ii) } u^{U}=u^{J}, q^{U}=q^{J}, p^{U}=p^{J}, \sigma^{U}=\sigma^{J} \text { for } r=R+H_{1} \text {, } \\
& \text { iii) } \quad u=0, p=0, q=Q \quad \text { for } r=R+H_{1}+H_{2} \text {. }
\end{aligned}
$$

Matching conditions in eq. (18) represent continuity requirements for the displacement of the solid matrix, the mass flux of fluid, pore pressure and the effective stress at the interface between the two rings.

The conditions prescribed in eq. (17) and (19) serve as the boundary conditions at the inner and outer boundaries for a single ring model of the trabecular meshwork.

\subsection{Dimensionless scheme}

The governing eqns. (14)-(16) and boundary conditions (17)-(19) are converted into the normalized form using the following nondimensional variables.

$\bar{p}=\frac{p}{2 \mu+\lambda}, \bar{r}=\frac{r}{R}, \bar{u}=\frac{u}{R}, \bar{q}=\frac{q}{Q}$,

$\overline{h_{1}}=\frac{H_{1}}{R}, \overline{h_{2}}=\frac{H_{2}}{R}, \bar{\sigma}_{v}^{\prime}=\frac{\sigma_{v}^{\prime}}{2 \mu+\lambda}$.

The non-dimensional equations and boundary conditions are as given below (dropping bars for convenience).

$$
\frac{d q}{d r}+\frac{q}{r}=0
$$

$\left(\frac{d^{2} u}{d r^{2}}+\frac{1}{r} \frac{d u}{d r}-\frac{u}{r^{2}}\right)-\frac{d p}{d r}=0$,

$\frac{d p}{d r}=\frac{\mu^{f} R Q}{(2 \mu+\lambda) k_{0}}\left[M\left(\frac{d u}{d r}+\frac{u}{r}\right)-1\right] q$.

and

i) $\quad \sigma_{v}^{\prime}=0 \quad$ for $r=1$,

ii) $\quad u^{U}=u^{J}, q^{U}=q^{J}, p^{U}=p^{J}, \sigma^{U}=\sigma^{J}$

iii) $\quad u=0, p=0, q=1 \quad$ for $r=1+h_{1}+h_{2}=R_{2}($ say $)$.

Using eq. (10) and (13) in eq. (9) the resulting relation in dimensionless form is given below:

$\sigma_{v}^{\prime}=\frac{d u}{d r}+\left(\frac{\lambda}{2 \mu+\lambda}\right) \frac{u}{r}$.

\subsection{Solution to the model}

Integrating eq. (20) we have,

$q=\frac{C_{1}}{r}$,

where $C_{1}$ is an integration constant. Now substitution of eqns. (22) and (27) into eq. (21) leads to the following nonhomogeneous differential equation with variable coefficients:

$r^{2} \frac{d^{2} u}{d r^{2}}+B r \frac{d u}{d r}-C u=-\kappa r$

where the group parameters,

$$
\begin{aligned}
B & =1-\frac{M \mu^{f} R Q C_{1}}{(2 \mu+\lambda) k_{0}}, C=1+\frac{M \mu^{f} R Q C_{1}}{(2 \mu+\lambda) k_{0}}, \\
\kappa & =\frac{\mu^{f} R Q C_{1}}{(2 \mu+\lambda) k_{0}} r .
\end{aligned}
$$

The solution of eq. (28) can be found as the sum of the general solution of the homogeneous equation (of the Cauchy-Euler type), $u_{h}$, and of a particular solution of the non-homogeneous equation, $u_{n}$ (see, e.g., [19]). The solution is given by 
$u=u_{h}+u_{n}$,

$u=C_{2}(r)^{v}{ }^{1}+C_{3}(r)^{v}{ }^{2}-\frac{\kappa}{B-C} r$

where $C_{2}$ and $C_{3}$ are integration constants, and $v_{1}$ and $v_{2}$ are the roots of the algebraic equation $v^{2}+(B-1) v-C=0$.

Using solutions (27) and (30) in eq. (22) and then integrating the resulting equation, we finally obtain the expression for the radial distribution of the fluid pressure:

$$
p=\frac{\mu^{f} R Q C_{1}}{(2 \mu+\lambda) k_{0}}\left[\begin{array}{c}
-\left(1+\frac{2 \kappa M}{B-C}\right) \ln r \\
+M C_{2} \frac{v_{1}+1}{v_{1}-1} r^{v_{1}-1} \\
+M C_{3} \frac{v_{2}+1}{v_{2}-1} r^{v_{2}-1}
\end{array}\right]+C_{4},
$$

where $C_{4}$ is the fourth integration constant of the boundary value problem.

Subjecting the expressions of $q, u$ and $p$ in eqns. (27), (30) and (31), respectively, to the boundary and matching conditions, the integration constants are determined and are given below:

\subsubsection{For single ring model}

$$
\begin{aligned}
& C_{1}=R_{2} \text {, } \\
& C_{2}=\left(\frac{\kappa}{B-C}\right) R_{2}^{1-v_{1}}-V_{0} R_{2}^{v_{2}-v_{1}} \text {, } \\
& C_{3}=\frac{2(\mu+\lambda)-\left\{v_{1}(2 \mu+\lambda)+\lambda\right\} R_{2}^{1-v_{1}}}{\left\{v_{2}(2 \mu+\lambda)+\lambda\right\}-\left\{v_{1}(2 \mu+\lambda)+\lambda\right\} R_{2}^{v_{2}-v_{1}}} \\
& \cdot\left(\frac{\kappa}{B-C}\right)=V_{0}(s a y) \\
& C_{4}=\left(1+\frac{2 \kappa M}{B-C}\right) \ln R_{2}-M\left[\begin{array}{l}
\left(\frac{\kappa}{B-C}\right) R_{2}^{1-v_{1}} \\
-V_{0} R_{2}^{v_{2}-v_{1}}
\end{array}\right] \frac{v_{1}+1}{v_{1}-1} R_{2}^{v_{1}-1} \\
& -M V_{0} \frac{v_{2}+1}{v_{2}-1} R_{2}^{v_{2}-1} \text {. }
\end{aligned}
$$

\subsubsection{For double ring model}

$$
C_{1}^{U}=C_{1}^{J}=R_{2},
$$

$$
C_{2}^{U}=\frac{1}{A_{22}}\left(A_{18}-V_{1} A_{23}\right),
$$

$$
C_{2}^{J}=\frac{1}{A_{3}}\left(A_{5}-V_{2} A_{4}\right),
$$

$$
C_{3}^{U}=\frac{A_{22}}{A_{17} A_{22}-A_{16} A_{23}}\left[\begin{array}{l}
V_{2}\left(A_{20}-\frac{A_{19} A_{4}}{A_{3}}\right) \\
+\frac{A_{19} A_{5}}{A_{3}}-\frac{A_{16} A_{18}}{A_{22}} \\
+A_{18}-A_{21}
\end{array}\right]=V_{1} \text { (say), }
$$

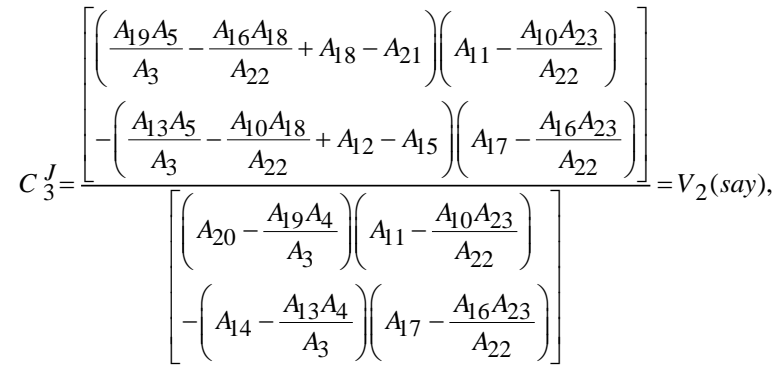

$$
\begin{aligned}
C_{4}^{U}=\left(\frac{A_{6} A_{23}}{A_{22}}-A_{7}\right) V_{1} & -V_{2}\left[\frac{A_{4}\left(A_{8}-A_{1}\right)}{A_{3}}-A_{9}+A_{2}\right] \\
& -\frac{A_{6} A_{18}}{A_{22}}+\frac{A_{5}\left(A_{8}-A_{1}\right)}{A_{3}},
\end{aligned}
$$

$C_{4}^{J}=\frac{A_{1}}{A_{3}}\left(V_{2} A_{4}-A_{5}\right)-V_{2} A_{2}$,

where

$A_{1}=\frac{M^{J} \mu^{f} R Q R_{2}}{\left(2 \mu^{J}+\lambda^{J}\right) k_{0}^{J}} \frac{v_{1}^{J}+1}{v_{1}^{J}-1}\left(R_{2}\right)^{v_{1}^{J}-1}$

$A_{2}=\frac{M^{J} \mu^{f} R Q R_{2}}{\left(2 \mu^{J}+\lambda^{J}\right) k_{0}^{J}} \frac{v_{2}^{J}+1}{v_{2}^{J}-1}\left(R_{2}\right)^{v_{2}^{J}-1}$

$A_{3}=\left(R_{2}\right)^{v_{1}^{J}} \quad A_{4}=\left(R_{2}\right)^{v_{2}^{J}} \quad A_{5}=-\frac{R_{2}}{2 M^{J}}$

$A_{6}=\frac{M^{U} \mu^{f} R Q R_{2}}{\left(2 \mu^{U}+\lambda^{U}\right) k_{0}^{U}} \frac{v_{1}^{U}+1}{v_{1}^{U}-1}\left(R_{1}\right)^{v_{1}^{U}-1}$

$A_{7}=\frac{M^{U} \mu^{f} R Q R_{2}}{\left(2 \mu^{U}+\lambda^{U}\right) k_{0}^{U}} \frac{v_{2}^{U}+1}{v_{2}^{U}-1}\left(R_{1}\right)^{v_{2}^{U}-1}$

$A_{8}=\frac{M^{J} \mu^{f} R Q R_{2}}{\left(2 \mu^{J}+\lambda^{J}\right) k_{0}^{J}} \frac{v_{1}^{J}+1}{v_{1}^{J}-1}\left(R_{1}\right)^{v_{1}^{J}-1}$

$A_{9}=\frac{M^{J} \mu^{f} R Q R_{2}}{\left(2 \mu^{J}+\lambda^{J}\right) k_{0}^{J}} \frac{v_{2}^{J}+1}{v_{2}^{J}-1}\left(R_{1}\right)^{v_{2}^{J}-1}$

$A_{10}=\left(R_{1}\right)^{v_{1}^{U}} \quad A_{11}=\left(R_{1}\right)^{v_{2}^{U}} \quad A_{12}=-\frac{R_{1}}{2 M^{U}}$

$A_{13}=\left(R_{1}\right)^{v_{1}^{J}} \quad A_{14}=\left(R_{1}\right)^{v_{2}^{J}} \quad A_{15}=-\frac{R_{1}}{2 M^{J}}$

$A_{16}=\left[\left(2 \mu^{U}+\lambda^{U}\right) v_{1}^{U}+\lambda^{U}\right]\left(R_{1}\right)^{v_{1}^{U}-1}$ 


$$
\begin{aligned}
& A_{17}=\left[\left(2 \mu^{U}+\lambda^{U}\right) v_{2}^{U}+\lambda^{U}\right]\left(R_{1}\right)^{v_{2}^{U}-1,} \\
& A_{18}=-\frac{\mu^{U}+\lambda^{U}}{M^{U}}, \quad A_{19}=\left[\left(2 \mu^{J}+\lambda^{J}\right) v_{1}^{J}+\lambda^{J}\right]\left(R_{1}\right)^{v_{1}^{J}-1} \\
& A_{20}=\left[\left(2 \mu^{J}+\lambda^{J}\right) v_{2}^{J}+\lambda^{J}\right]\left(R_{1}\right)^{v_{2}^{J}-1}, \quad A_{21}=-\frac{\mu^{J}+\lambda^{J}}{M^{J}}, \\
& A_{22}=\left[\left(2 \mu^{U}+\lambda^{U}\right) v_{1}^{U}+\lambda^{U}\right], \\
& A_{23}=\left[\left(2 \mu^{U}+\lambda^{U}\right) v_{2}^{U}+\lambda^{U}\right]
\end{aligned}
$$

\section{Results and discussion}

The small-strain solutions given by eqns. (27), (30), and (31) may be used to quantify the hydro mechanical behavior of the TM. The elastic properties of the material are specified by the Young's modulus, E, and Poisson's ratio, $v$. The lame constants $\mu$ and $\lambda$, defining the elastic properties of the meshwork, are related to the parameters by:

$$
\mu=E /[2(1+v)], \quad \lambda=E v /[(1+v)(1-2 v)] .
$$

\begin{tabular}{|c|c|c|}
\hline $\begin{array}{l}\text { Control } \\
\text { Parameters }\end{array}$ & Description & $\begin{array}{c}\text { Typical } \\
\text { physiological value* }\end{array}$ \\
\hline $\mathrm{R}$ & Radius of the anterior chamber & $5.62 \times 10^{-3} \mathrm{~m}$ \\
\hline Q & $\begin{array}{l}\text { Characteristic velocity of } \\
\text { aqueous fluid }\end{array}$ & $3.03 \times 10^{-6} m s^{-1}$ \\
\hline $\mathrm{E}$ & Elastic modulus of the meshwork & $4.5 \times 10^{5} N m^{-2}$ \\
\hline$v$ & Poisson's ratio & 0.35 \\
\hline M & Material constant & -4.5 \\
\hline $\mathrm{H}$ & $\begin{array}{l}\text { Thickness of the trabecular } \\
\text { meshwork }\end{array}$ & $0.01 \times 10^{-3} \mathrm{~m}$ \\
\hline$H_{1}$ & $\begin{array}{l}\text { Thickness of the uveal- } \\
\text { corneoscleral meshwork }\end{array}$ & $7.868 \times 10^{-5} \mathrm{~m}$ \\
\hline$H_{2}$ & $\begin{array}{l}\text { Thickness of the juxtacanalicular } \\
\text { meshwork }\end{array}$ & $2.248 \times 10^{-5} \mathrm{~m}$ \\
\hline$\mu^{f}$ & $\begin{array}{l}\text { Dynamic viscosity of aqueous } \\
\text { humor }\end{array}$ & $1.0 \times 10^{-3}$ Pas \\
\hline$k_{0}$ & $\begin{array}{l}\text { Permeability of the trabecular } \\
\text { meshwork }\end{array}$ & $1.99 \times 10^{-12} m^{2}$ \\
\hline$k_{0}^{J}$ & Permeability of the JCM & $1.99 \times 10^{-13} \mathrm{~m}^{2}$ \\
\hline$k_{0}^{U}$ & Permeability of the UCM & $1.99 \times 10^{-11} m^{2}$ \\
\hline
\end{tabular}

Computational results have been obtained for the proposed model by introducing estimated values of the physiological parameters listed in Table 1.

Table 1:

*Estimated and used by Tandon and Avtar [23].
The distribution of solid displacement in the trabecular meshwork has been displayed in Fig.3. It is observed from the graph that the displacement decreases along radial distance. The decrease in the displacement in the UCM is insignificant whereas the displacement decrease sharply in the JCM. The outflow of aqueous humor through the trabecular meshwork occurs under the pressure differential across the meshwork. The interaction between the flowing aqueous humor and the solid matrix of the porous meshwork gives rise deformation in the matrix. The juxtacanalicular meshwork is structurally stiffer than the uveal-corneoscleral meshwork. The displacements in solid matrix near the AC surface are higher than that away the AC surface. The above observation is supported by this fact. The change in solid displacement for a single ring model is relatively very small as compared to the same for a double ring model.

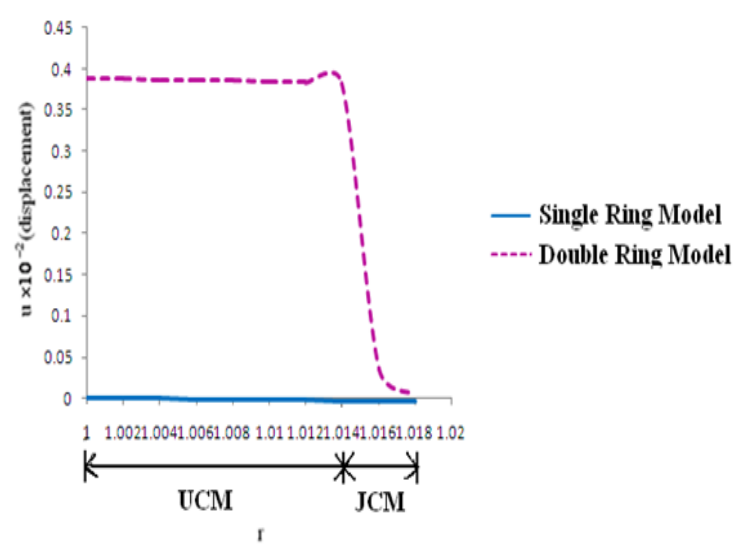

Fig. 3: Radial Displacement vs. Distance for Single and Double Ring Model on Displacement

The effects of two structural parameters in the model: the Poisson's ratio and elastic modulus on the solid displacement have been depicted in Fig.4(A) and Fig.4(B), respectively. As is evident from the curves in these figures, an increase in both the Poisson's ratio and elastic modulus reduces the solid displacement. An increase in the Poisson's ratio is caused by an increase in lateral contraction or a decrease in elongation in the direction of the applied strain or by both. Therefore, an increase in the Poisson's ratio reduces the displacement.

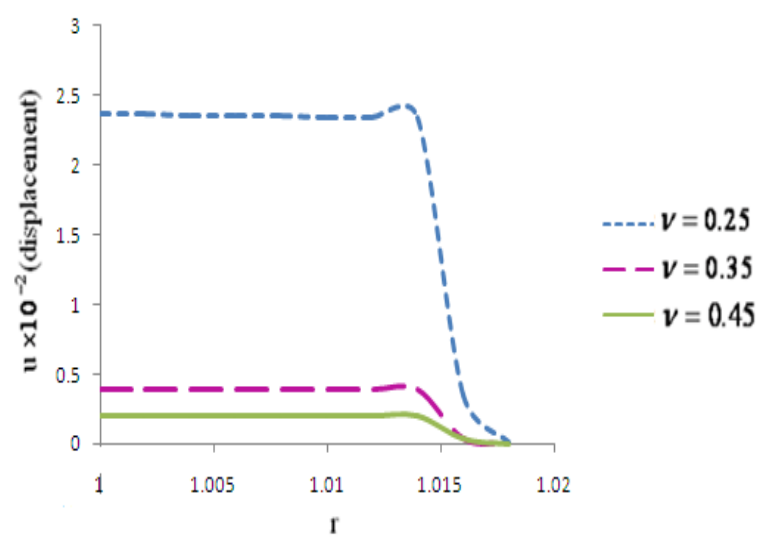

Fig. 4(A): Radial Displacement vs. Distance from AC Surface for Different Poisson's Ratios. 
As is observed from the curves in Fig.4(B), the displacement is reduced with an increase in the elastic modulus of the meshwork. Due to a higher elastic modulus, the matrix offers a higher resistance to being it deformed which results in a decreased displacement in the solid phase.

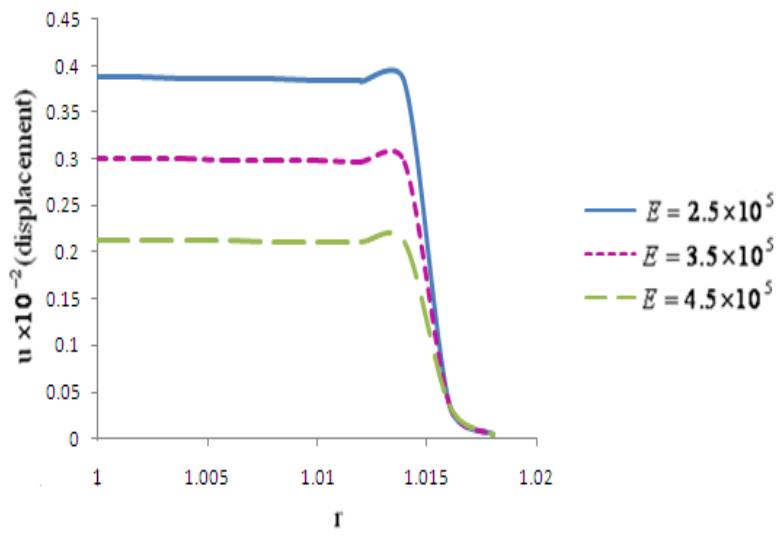

Fig. 4(B): Radial Displacement vs. Distance from AC Surface for Different Elastic Moduli.

It is evident from the curves in Fig.4(C) that the solid displacement in the trabecular tissue is reduced with a reduction in the value of material constant $\mathrm{M}$. A decrease in the values of material constant $\mathrm{M}$ increases the permeability of the meshwork which facilitates the outflow in the meshwork. This results in reduced displacement.

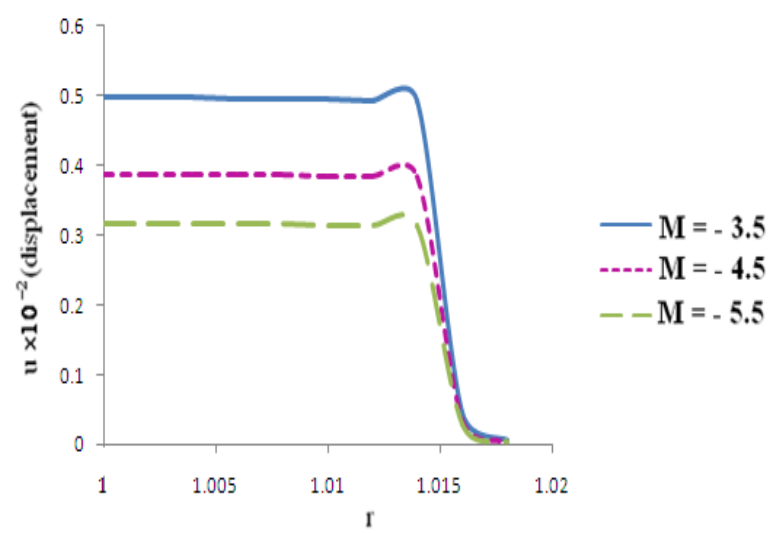

Fig. 4(C): Radial Displacement vs. Distance from AC Surface for Different Values of Material Constant.

The distribution of dilatation which represents aqueous humor fluid content in the trabecular meshwork has been shown in Fig.5. It is evident from the curves in Fig.5 that dilatation of the ocular tissue decrease sharply along radial displacement from the anterior chamber towards the canal of Schlemm. The UCM is highly porous structure and the JCM is less porous. The content of aqueous humor in the UCM is more than that in the JCM. The dilatation decreases with decrease in the porosity along the radial distance. The change in dilatation for the single ring model is insignificant as compared to the same for the double ring model.

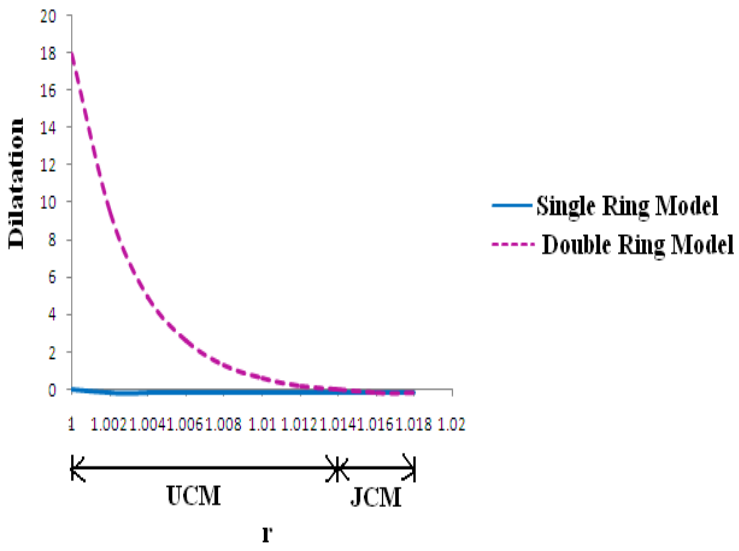

Fig. 5: Dilatation vs. Distance for Single and Double Ring Model.

The effects of Poisson's ratio and elastic modulus of the meshwork on the dilatation have been shown in Figs.6(A) and 6(B) respectively. An increase in both the Poisson's ratio and elastic modulus cause decrease in the radial displacement which result decrease in the dilatation i.e. the change in the fluid content.

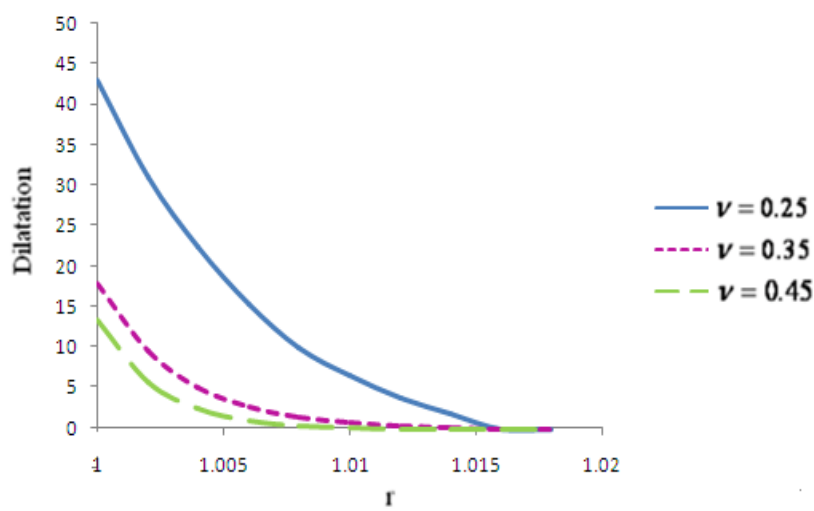

Fig. 6(A): Dilatation vs. Distance from AC Surface for Different Poisson's Ratios.

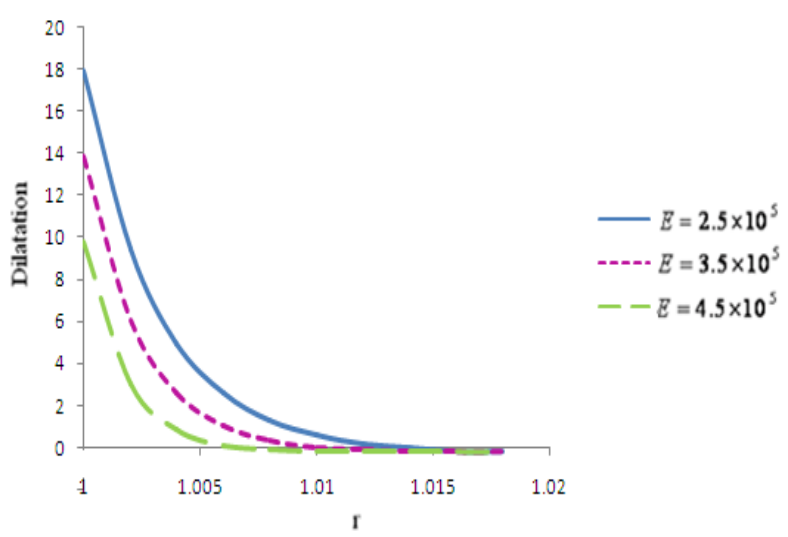

Fig. 6(B): Dilatation vs. Distance from AC Surface for Different Elastic Moduli.

It is obvious from the curves in Fig.6(C) that the decrease in the material constant decreases the dilatation in the tissue. A decrease with value of material constant enhances the matrix permeability which facilitates the aqueous outflow. This reduces deformation/displacement in the solid phase causing a decrease in the dilatation. 


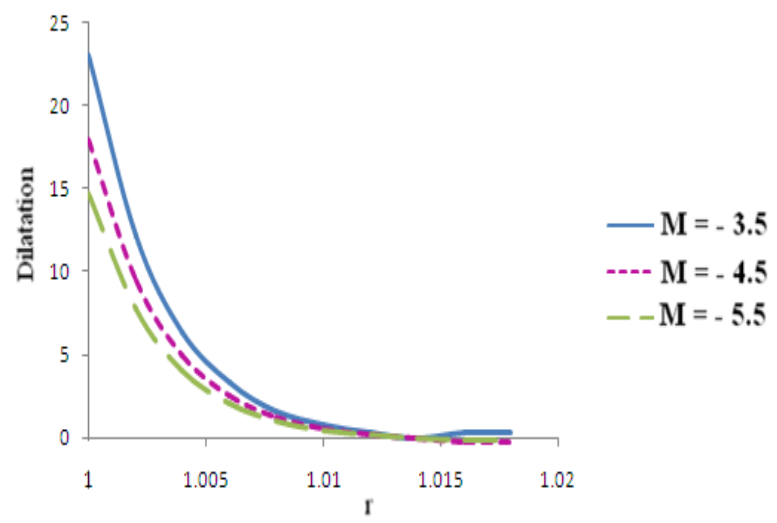

Fig. 6(C): Dilatation vs. Distance from AC Surface for Different Values of Material Constant.

\section{Conclusion}

The prime objective of the present work was to develop a simple mathematical model for describing the outflow of aqueous humor in the porous trabecular meshwork of eye and to conduct a theoretical analysis of the sensitivity of the outflow characteristics of aqueous humor in the trabecular meshwork to its structural properties. As an advantage of the analytic approach of the present work, the explicit mathematical expressions were derived to represent and to predict physical results of the fluid dynamics aspects of aqueous humor outflow. The computational results obtained by using simplified geometry and rough estimates of material properties illustrate that the salient biomechanical features of the aqueous humor outflow result from the response of the trabecular meshwork to well understood mechanical forces. The model incorporates the interaction between the aqueous humor outflow and the solid matrix of the porous medium. The interaction, representing the mechanical loading on the matrix gives rise to the deformations.

An examination of the analytic results, presented through the graphs reveal that the radial displacement (i.e. deformation) and dilatation in the TM matrix are maximum in the inlet region (the vicinity of anterior chamber) and continuously decreases to reach zero at the outlet boundary. The displacement and dilatation are decreased with an increase in both the structural parameters: the Poison's ratio and elastic modulus, whereas an increase in the material constant increases both the deformation and the dilatation.

\section{References}

[1] R.J. Atkin and R.E. Craine, Continuum theories of mixtures: applications, Inst. Math. Appl. 17 (1976) 153.http://dx.doi.org/10.1093/imamat/17.2.153.

[2] R. Avtar, R. Srivastava, Aqueous outflow in Schlemm's canal, Appl. Math. Comput. $174 \quad$ (2006) 316328.http://dx.doi.org/10.1016/j.amc.2005.04.081.

[3] R. Avtar, R. Srivastava, Modelling aqueous humor outflow through trabecular meshwork, Appl. Math. Comput. 189 (2007) 734 745.http://dx.doi.org/10.1016/j.amc.2006.11.109.

[4] R.M. Bowen, Theory of mixtures in Continuum Physics, A.C Eringen (Ed), Vol. II, New York: Academic (1976).

[5] P.A. Chandler, W.M. Grant, Glaucoma, second ed.,Lea\&Febiger, Philadelphia, 1979, pp. 77-109.

[6] C.R. Ethier, R.D. Kamm, B.A. Palaszewski, M.C. Johnson, T.M Richardson, Calculations of flow resistance in the juxtacanalicular meshwork, Invest Ophthalmol. Vis. Sci. (1986) 27(12) 1741-1750.

[7] C.R. Ethier, M. Johnson, J. Rubert, Ocular biomechanics and biotransport, Ann. Rev. Biomed. Eng. 6 (2004) 249 273.http://dx.doi.org/10.1146/annurev.bioeng.6.040803.140055.

[8] W.M. Grant, Facility of flow through the trabecular meshwork, Arch. Ophthalmol. $54 \quad$ (1955) 248.http://dx.doi.org/10.1001/archopht.1955.00930020251012.
[9] W.M. Grant, Further studies on facility of flow through the trabecular meshwork, Arch. Ophthalmol. 60 (1958) 523533.http://dx.doi.org/10.1001/archopht.1958.00940080541001.

[10] M. Johnson, Modulation of outflow resistances by the pores of the inner wall endothelium, Invest. Ophth. Vis. Sci. 33 (5) (1992) 1670-1675

[11] D.E. Kenyon, Transient filtration in a porous cylinder, Appl. Mech 43 (1976) 594-598.http://dx.doi.org/10.1115/1.3423938.

[12] M. Klanchar, J.M. Tarball, Modelling water flow through arterial tissue, Bull. Math. Bio. $49 \quad$ (1987) 651669.http://dx.doi.org/10.1007/BF02481766.

[13] W.M. Lai, V.C. Mow, Drag induced compression of articular cartilage during a permeation experiment, Biorheology 17 (1980) 111123.

[14] A. Llobet, X. Gasull, A. Gual, Understanding trabecular meshwork physiology: a key to the control of intraocular pressure, News Physiol. Sci. 18 (2003) 205-209.

[15] W.K. McEwen, Application of Poiseuille's law of aqueous outflow, Arch. Ophthalmol, $60 \quad$ (1958) 307.http://dx.doi.org/10.1001/archopht.1958.00940080306017.

[16] R.A. Moses, The effect of intraocular pressure on resistance of outflow, Surv. Ophthalmol. $22 \quad$ (1977) 88 100.http://dx.doi.org/10.1016/0039-6257(77)90088-1.

[17] R.A. Moses, Circumferential flow in Schlemm's Canal, Am. J. Ophthalmol. 88 (1979) 585-591.http://dx.doi.org/10.1016/00029394(79)90519-1.

[18] R.A. Moses, Jr. Wj. Grodzki, EL. Etheridge, C.D. Wilson, Schlemm's canal: The effect of intraocular pressure, Invest. Ophthalmol Vis. Sci. Vol. 20(1) (1981) 61-68.

[19] A.L. Rabenstain, Introduction to Ordinary Differential Equations, New York: Academic (1972).

[20] A.J. Sit, Hydrodynamics of aqueous humor outflow, S.M. Thesis, Massachusetts Institute of technology, 1995.

[21] A.J. Sit, H. Gong, R. Nathan, F.T. Freddo, R. Kamm, M. Johnson, The role of soluble proteins in generating aqueous outflow resistance in the bovine and human eye, Exp, Eye Res. 64 (1997) 813 821.http://dx.doi.org/10.1006/exer.1997.0276.

[22] T. Seiler, J. Wollensak, The resistance of the trabecular meshwork to aqueous humor outflow, Graefes Arch. Clin. Exp. Ophthalmol. (1985) 223(2) 88-91.http://dx.doi.org/10.1007/BF02150951.

[23] P.N. Tandon, R. Avtar, Biphasic model of the trabecular meshwork in the eye, Med. Biol. Eng. Comput. 29 (1991) 281 290.http://dx.doi.org/10.1007/BF02446710. 